\title{
Doping Properties and Phase Transition in Single-Layer $\mathrm{MoS}_{2}$
}

Yung-Chang Lin ${ }^{1}$, Dumitru O. Dumcenco ${ }^{2}$, Hannu-Pekka Komsa ${ }^{3}$, Yoshiko niimi ${ }^{1}$, Arkady V. Krasheninnikov $^{3}$, Ying-Sheng Huang ${ }^{2}$, Kazu Suenaga ${ }^{1}$

1. National Institute of Advanced Industrial Science and Technology (AIST), Tsukuba 305-8565, Japan

2. Department of Electronic Engineering, National Taiwan University of Science and Technology, Taipei 10607, Taiwan

3. Department of Physics, University of Helsinki, P.O. Box 43, Fl-00014 Helsinki, Finland

Layered inorganic transition metal dichalcogenides (TMDs) exhibit wide variety of electronic properties [1]. Among them, single-layer molybdenum disulfide $\left(\mathrm{SL}-\mathrm{MoS}_{2}\right)$ is one of the most prominent TMDs, which is a direct bandgap semiconductor and possesses carrier mobility comparable to graphene nano-ribbon with high current on-off ratio [2]. The electrical conductivity of $\mathrm{MoS}_{2}$ can be further modulated by substitutional doping or adding adatoms. The knowledge of how does the dopant atom interact with the $\mathrm{MoS}_{2}$ lattices is highly important in the fundamental science and device applications. Here, we present the difference in the behavior of Re (n-type) and $\mathrm{Au}$ (p-type) dopant atoms in SL-MoS ${ }_{2}$ using in situ STEM [3]. 0.5 1 at \% of Re or Au doped $\mathrm{MoS}_{2}$ single-crystals were synthesized by the chemical vapor transport method. STEM imaging was carried out in an aberration-corrected STEM JEOL-2100F equipped with a DELTA corrector and a cold field emission gun operated at $60 \mathrm{kV}$. Figure 1 shows the ADF images of Re and Au doped SL-MoS 2 where Re atoms tend to substitute Mo atoms (Fig. 1a), while Au atoms exist as adatoms and migrate on $\mathrm{MoS}_{2}$ surface (Fig. 1b-1d). The atomic positions of dopants in $\mathrm{MoS}_{2}$ were confirmed by fitting the experimental ADF profiles with the simulated ones with possible specimen inclinations taken into account. The in situ experiment (Fig. 2) together with DFT calculations (Fig. 3) discovered that the Re dopant showed higher surface reactivity and enhanced chemical affinity, evidenced by agglomeration of Re atoms and other impurities such as carbon.

Polymorphism is one of the unique features in SL-MoS 2 . Depending on the arrangement of S atoms, SL-MoS $\mathrm{S}_{2}$ appears trigonal prismatic $(2 \mathrm{H})$ and octahedral (1T) symmetries with distinct electronic characteristics, where $2 \mathrm{H}$ is semiconducting and $1 \mathrm{~T}$ is metallic. We present the dynamical process of phase transition between $2 \mathrm{H}$ and $1 \mathrm{~T}$ phases in SL-MoS2 with atomic precision using in situ STEM [4]. The phase transition comprises of $\mathrm{S}$ and/or Mo atomic-plane gliding. A new phase ( $\alpha$ phase), the precursor of phase transition, consists of 3 4 constricted zigzag chains which was observed for the first time. Migration of two kinds of boundaries ( $\beta$ and $\gamma$-boundaries) is also found to be responsible for the growth of the second phase. The $1 \mathrm{~T}$ phase with variable size can be intentionally introduced in the $2 \mathrm{H}$ matrix by using a high dose of incident electron beam at high temperature $\left(400 \sim 700^{\circ} \mathrm{C}\right)$. This work may lead to the possible fabrication of composite nano-devices made of local domains with distinct electronic properties.

\section{References:}

[1] M. Chlowalla et al., Nat. Chem. 5 (2013) 263-275.

[2] Q. H. Wang et al., Nat. Nanotech. 7 (2012) 699-712.

[3] Y. C. Lin et al., Adv. Mater. in press, (2014).

[4] Y. C. Lin et al., submitted, (2014).

[5] This research is supported by a JST Research Acceleration Program. 

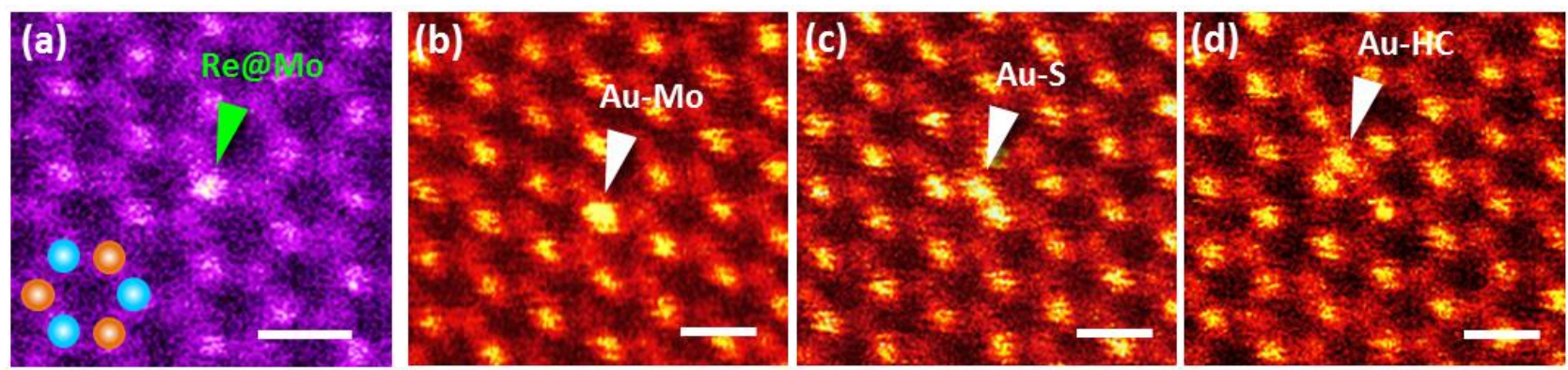

Figure 1. (a) The ADF image of Re-doped SL-MoS 2 . The Re substitution at Mo site (Re@Mo) is pointed by green arrow. Blue balls and orange balls indicate Mo and two overlapped S atoms. (b-d) The ADF images of Au-doped SL-MoS , where the Au adatom (indicated by white arrows) located on top of the Mo (Au-Mo), S (Au-S), and hallow center (Au-HC), respectively. Scale bar: $3 \AA$.
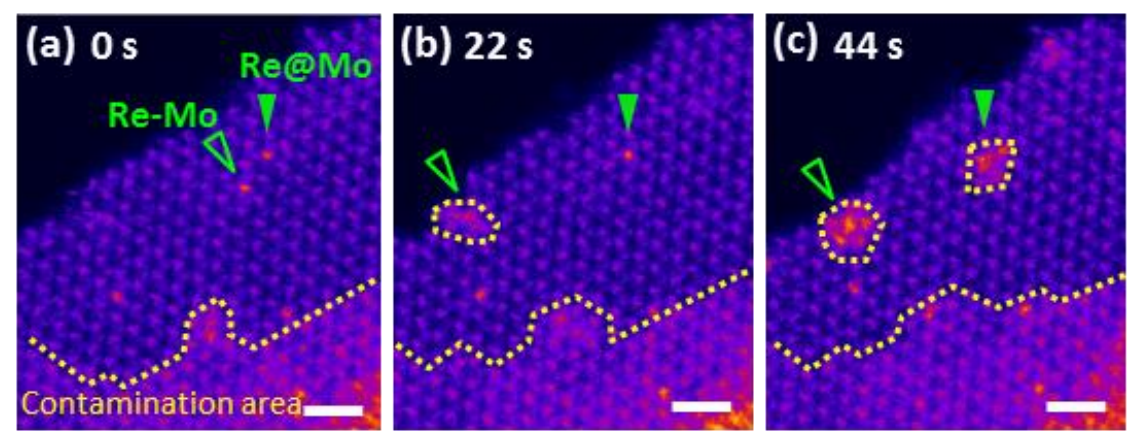

Figure 2. (a-c) Three sequential ADF images show the dynamic behavior of carbon contaminations on Re-doped SL-MoS 2 surface. The Re dopants are indicated by green arrows while the yellow dot lines highlight the carbon contaminated regions. Scale bar: $1 \mathrm{~nm}$.

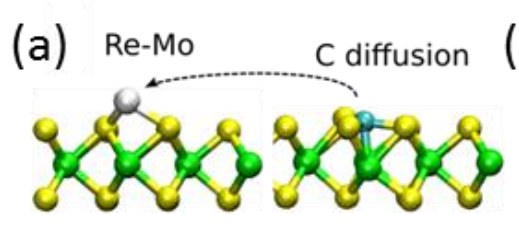

(b) Re@Mo
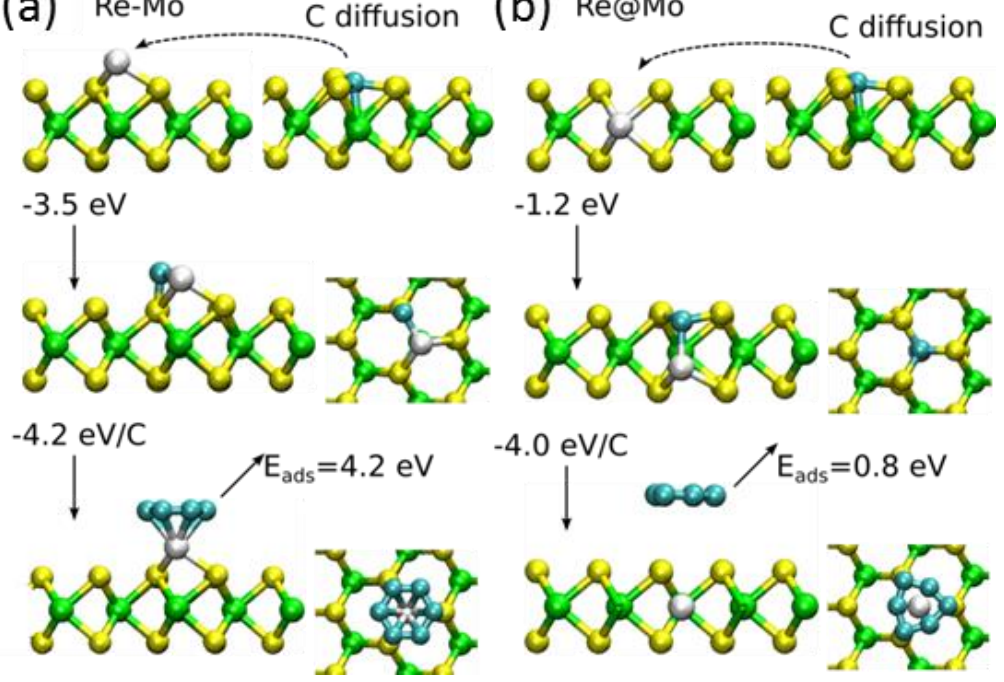

Figure 3. Illustration of the carbon segregation processes around (a) Re-Mo and (b) Re@Mo dopants. The related energies are obtained from DFT calculations. $E_{\text {ads }}$ corresponds to the energy required to remove the molecule from the surface. 